\title{
Optical effects of multipole interaction in aggregated structures: Rigorous scattering solution for a periodic chain of cylinders
}

\author{
Zhe Chen* and Ping Sheng \\ Corporate Research Science Laboratories, Exxon Research and Engineering Company, Annandale, New Jersey 08801
}

(Received 12 September 1988; revised manuscript received 23 December 1988)

\begin{abstract}
Recent experimental studies on the optical properties of gold colloidal aggregates have found interesting characteristics, such as double absorption peaks and enhanced depolarized scattering, that are not explainable in terms of traditional mean-field theories. Based on the observation that the aggregate clusters have a locally chainlike structure of touching spheres, a simple model for the calculation of their absorption and scattering characteristics is proposed that consists of a randomly oriented chain of cylindrical particles under the excitation of an electromagnetic field. The consideration of cylindrical geometry considerably simplifies the mathematics while retaining the essential physics of capacitive coupling between the particles. In the optical frequency regime, a rigorous scattering solution for the model shows that, besides the single-particle Mie resonance, there exists a low-frequency collective excitation arising from the anisotropic, high-multipole interactions between the metal particles. It results in a second absorption peak whose peak position is controlled by the capacitive coupling between the adjacent particles. Both the polarized and the depolarized scattering also show accompanying increases at the collective excitation frequency. Good agreement is found between the calculated results and the experimental observations.
\end{abstract}

\section{INTRODUCTION}

The study of the optical properties of particulate aggregates is of great importance in many diverse areas of science and technology ranging from absorption and scattering of light by interstellar dust ${ }^{1,2}$ to the optical effects of air and water pollutants. Of essential importance in determining the optical properties of such aggregates is a detailed knowledge of their structure. Progress has been achieved recently with the realization that aggregates often possess scale-invariant structures. ${ }^{3}$ Indeed, light scattering has been widely used to determine the fractal dimension $d_{f}$ of aggregates, ${ }^{4-6}$ since in the single-scattering approximation the structure factor $S(q) \sim q^{-d_{f}}$, where $q$ is the scattering wave vector. In a separate publication, ${ }^{7}$ we have addressed the important question of the effects of multiple scattering on the structure factor and found that the dominant effect of multiple scattering is reflected in the mean-field index of refraction of the clusters and therefore does not mask the $q$ dependence of the scattering. However, it was noted that the traditional mean-field treatments, such as the MaxwellGarnett theory, ${ }^{8}$ do not adequately describe the absorption of the clusters since they are based on the random, isotropic distribution of neighboring particles and thus ignore the strong short-range anisotropic correlations inherent in a connected cluster. For metallic clusters, a mean-field approach will therefore not be able to account for the experimental observations ${ }^{9}$ such as the appearance of a second, red-shifted absorption peak (other than the Mie-resonance peak), or the large degree of depolarized scattering. We suggest that a more appropriate approach for these properties is through the calculation of the effective polarizability of small chainlike structures as seen from the transmission electron microscope (TEM) pictures of these clusters, subjected to an average field determined by the mean-field index of refraction.

In this work we will focus our attention on the absorption and depolarized scattering characteristics of gold colloidal aggregates whose structures have been extensively characterized. ${ }^{10}$ Since the metal particles (diameter $d \sim 15 \mathrm{~nm}$ ) in the clusters are in physical contact with their nearest neighbors, it is important to first assess the nature of their electromagnetic coupling by estimating the effective $R C$ time constant. Transmission electron micrographs show that the metal spheres are connected to their neighbors by a metal bond with a width $w$ that is at most a few angstroms. Since this constriction is substantially less than the bulk mean free path of the electrons, the effective resistance between the particles is expected to be increased substantially. ${ }^{11}$ If we account for this by $^{7} \rho=\rho_{0}(1+l / w)$, where $\rho_{0}$ is the bulk resistivity and $l$ the bulk mean free path, then the resistance $R$ of the contact would be $R \sim \rho l / w^{2}$. For the capacitance $C$, we note that the capacitance of two spheres that are touching is $d \ln 2$, where $d$ is the diameter of the spheres. Using $w \sim 0.03 d(\sim 5 \AA)$, we get $\omega R C \sim 10^{2} \gg 1$ at optical frequencies. It follows that the coupling is predominantly capacitive in nature.

To capture the effects of strong high-multipole interaction between the metal particles, we propose a simple model of a periodic chain of cylindrical metallic segments under the excitation of an electromagnetic field. The consideration of cylindrical geometry enables an easy formulation of an eigenvalue problem for the electromagnetic field in the stratified cylindrical region. It is shown in the Appendix A that the eigenfields can be expressed explicitly in analytical form, so that mathematically the 
problem is as simple as that for the infinite homogeneous cylinder. Nonetheless, this simple model does reflect the essential physics of capacitive coupling between the particles. An alternative approach would be to consider the scattering solution for two or more touching spheres. ${ }^{12-16}$ However, the mathematics would then be significantly more complex. One may view the net effect of our model calculation as the renormalization of the metal-particle polarizability $\alpha$ due to the high-multipole interactions with the neighbors. The renormalized $\alpha$ would be a tensorial quantity with uniaxial symmetry, where the symmetry axis coincides with the local chain axis. Rigorous scattering solution shows that when the incident wave is polarized along the symmetry direction, the renormalized $\alpha$ exhibits a second, red-shifted absorption peak as distinct from the single-particle Mie resonance. The depolarized scattering also shows dramatic increase around this absorption peak frequency. Averaging over random orientation of the chain yields the double absorption peaks and the enhanced depolarized scattering that are in good agreement with experimental observations. In what ensues, formulation of our solution approach for the periodic chain is given in Sec. II. This is followed by a discussion of numerical results and their comparison with experiments in Sec. III. Section IV concludes with remarks noting some problems in our approach as well as further research opportunities. Some of the more lengthy mathematical derivations are given in appendixes.

\section{FORMULATION}

Our goal in this section is to present a relatively straightforward, but rigorous, solution of Maxwell's equations for a periodic chain of cylindrical particles illuminated by an incident plane wave $\mathbf{E}_{I}$ $=\mathbf{E}_{0} \exp \left(j \mathbf{k}_{0} \cdot \mathbf{r}-j \omega t\right)$, where $\mathbf{k}_{0}$ is the wave vector, $\omega$ is the frequency, and $t$ is time. The chain is embedded in a matrix with a dielectric constant $\epsilon_{0}$. As shown in Fig. 1,

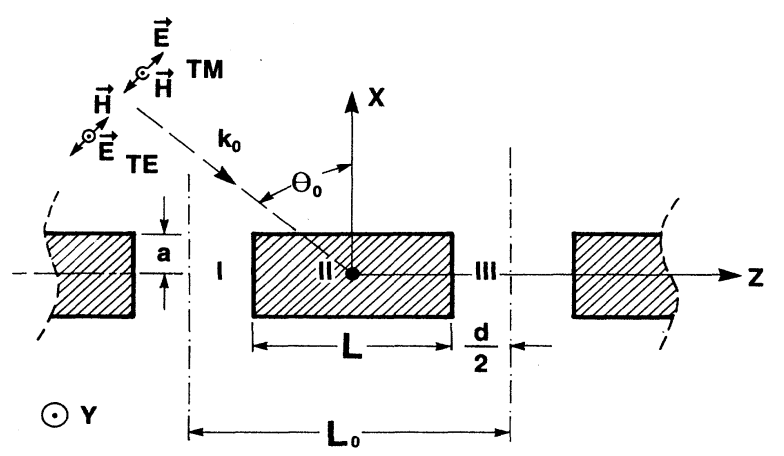

FIG. 1. Sketch of a portion of the periodic chain of cylinders. Parameters for the cylinders are radius $a$, length $L$, separation $d$, and periodicity $L_{0}$. The cylindrical coordinate system is chosen such that the $r \phi$ plane coincides with the $x y$ plane and the counterclockwise direction represents the positive direction of $\widehat{\mathbf{e}}_{\phi}$. In the TM (TE) mode the electric field is polarized in (perpendicular to) the incident plane $x z$. the length of each cylindrical segment is denoted by $L$ and the radius of the cross section by $a$. If the separation between two adjacent cylinders is $d$, then the periodicity is $L_{0}=L+d$. The dielectric constant of the particles is denoted by $\epsilon(\omega)$, and magnetic susceptibility $\mu=1$. Instead of following the traditional approach, i.e., solving the Maxwell equations in each homogeneous region and matching the boundary conditions at interfaces, we employ the periodicity in the problem to construct an eigenvalue problem for the electromagnetic excitations in the region $r \leq a$. The electromagnetic field in the $r \leq a$ region is then expanded in terms of these eigenfields, each of which now rigorously satisfies the boundary conditions at internal periodic interfaces. The expansion coefficients of these excited fields, as well as the scattering waves, are then determined by matching the boundary conditions at the single interface $r=a$.

\section{A. General solutions of Maxwell equations}

In a linear, isotropic, and homogeneous medium, the sourceless time harmonic electromagnetic fields satisfy the following equations:

$$
\begin{aligned}
& \nabla^{2} \mathbf{E}+k^{2} \mathbf{E}=0, \\
& \nabla^{2} \mathbf{H}+k^{2} \mathbf{H}=0, \\
& \nabla \cdot \mathbf{E}=0, \\
& \nabla \cdot \mathbf{H}=0,
\end{aligned}
$$

where $\mathbf{E}$ and $\mathbf{H}$ are the spatial-dependent electric- and magnetic-field vectors, respectively, and $k$ is the propagation wave vector obeying the dispersion relation $k^{2}=\omega^{2} \epsilon_{0}$. Here $\epsilon_{0}$ is the dielectric constant of the medium. The $\mathbf{E}$ and $\mathbf{H}$ are related to each other by

$$
\begin{aligned}
& \boldsymbol{\nabla} \times \mathbf{E}=j \omega \mathbf{H}, \\
& \boldsymbol{\nabla} \times \mathbf{H}=-j \omega \epsilon_{0} \mathbf{E} .
\end{aligned}
$$

It is straightforward to show ${ }^{17}$ that Eq. (1) can be satisfied by the functions $\mathbf{M}$ and $\mathbf{N}$, where

$$
\begin{aligned}
& \mathbf{M}=\boldsymbol{\nabla} \times(\mathbf{c} \psi), \\
& \mathbf{N}=\boldsymbol{\nabla} \times \mathbf{M} / k,
\end{aligned}
$$

c is an arbitrary constant vector, and $\psi$ is a scalar function satisfying the scalar Helmholtz equation

$$
\nabla^{2} \psi+k^{2} \psi=0
$$

The vector functions $\mathbf{M}$ and $\mathbf{N}$, generally denoted as vector harmonics, therefore have all the required properties of the electromagnetic fields. The form of generating function $\psi$ is dictated by the symmetry of the problem. For cylindrical symmetry,

$$
\psi_{n}(r, \phi, z)=R_{n}(\rho) e^{j n \phi} e^{j h z},
$$

where $R_{n}(\rho)$ is a Bessel function of order $n, h$ is a separation constant to be determined, and $\rho=r\left(k^{2}-h^{2}\right)^{1 / 2}$. The vector harmonics $\mathbf{M}, \mathbf{N}$ are obtained from Eq. (2) and by letting $\mathbf{c}=\widehat{\mathbf{e}}_{z}$ : 


$$
\begin{aligned}
& \mathbf{M}_{n}=\left\{j n u\left[R_{n}(\rho) / \rho\right] \widehat{\mathbf{e}}_{r}-u R_{n}^{\prime}(\rho) \widehat{\mathbf{e}}_{\phi}\right\} e^{j n \phi} e^{j h z}, \\
& \mathbf{N}_{n}=\frac{1}{k} \nabla \times \mathbf{M}_{n},
\end{aligned}
$$

where $u=\left(k^{2}-h^{2}\right)^{1 / 2} . \mathbf{M}_{n}$ and $\mathbf{N}_{n}$ obtained above are noted to be orthogonal:

$$
\int_{0}^{2 \pi} \mathbf{M}_{n} \cdot \mathbf{M}_{n}^{*} d \phi=\int_{0}^{2 \pi} \mathbf{N}_{n} \cdot \mathbf{N}_{n}^{*} d \phi=\int_{0}^{2 \pi} \mathbf{M}_{n} \cdot \mathbf{N}_{n}^{*} d \phi=0,
$$

where the asterisk represents complex conjugation.

The incident and scattered waves can now be expanded in terms of the above vector harmonics with $R_{n}(\rho)$ and $h$ determined as follows. For the incident waves, we distinguish between two polarizations as shown in Fig. 1. In case (1) an incident wave is polarized in the incident plane, denoted as the TM mode, and in case (2) the wave is polarized perpendicular to the incident plane, denoted as the TE mode. An arbitrary incident wave can be decomposed into a linear combination of these two independent cases.

In the case (1), we write the $E$ field of the incident wave as

$$
\mathbf{E}_{I}^{(1)}=\left(\sin \theta_{0} \widehat{\mathbf{e}}_{x}+\cos \theta_{0} \widehat{\mathbf{e}}_{z}\right) E_{0} e^{-j k_{0}\left(x \cos \theta_{0}-z \sin \theta_{0}\right)} .
$$

Since $\mathbf{E}_{I}$ is finite at $r=0, R_{n}(\rho)$ in Eqs. (4) and (5) must be the Bessel function of first kind, $J_{n}(\rho)$. It is clear from Eq. (7) that the $z$ dependence of the incident wave is explicitly contained in the phase factor, and therefore the separation constant $h$ should be equal to $k_{0} \sin \theta_{0}$. The generating function for the incident wave is thus

$$
\psi_{n}^{(I)}(r, \phi, z)=J_{n}\left(k_{0} r \cos \theta_{0}\right) e^{j n \phi} e^{j k_{0} z \sin \theta_{0}} .
$$

Expanding Eq. (7) in terms of the vector harmonics that are generated by $\psi_{n}^{(I)}$, we have

$$
\begin{aligned}
& \mathbf{E}_{I}^{(1)}=\sum_{n} E_{n} \mathbf{N}_{n}^{(I)}, \\
& \mathbf{H}_{I}^{(1)}=\frac{1}{j k_{0}} \boldsymbol{\nabla} \times \mathbf{E}_{I}^{(1)}=-j \sum_{n} E_{n} \mathbf{M}_{n}^{(I)},
\end{aligned}
$$

where the expansion coefficient is

$$
E_{n}=\frac{(-j)^{n} E_{0}}{k_{0} \cos \theta_{0}}
$$

In case (2), $\mathbf{E}_{I}$ is polarized in the $y$ direction. The expansion in terms of the same vector harmonics yield $\mathbf{E}_{I}^{(2)}=\mathbf{H}_{I}^{(1)}$ and $\mathbf{H}_{I}^{(2)}=-\mathbf{E}_{I}^{(1)}$.

For the scattered waves, since they are propagating outward from $r=a, R_{n}(\rho)$ must be Henkel functions of first kind, $H_{n}^{(1)}(\rho)$, for which $H_{n}^{(1)}(\rho)$ $\rightarrow(2 / \pi \rho)^{-1 / 2} e^{j(\rho-n \pi / 2-\pi / 4)}$ as $\rho \rightarrow \infty$. Apart from a $z-$ dependent incident-wave phase factor, the scattering waves must be periodic in $z$ in order to fit the boundary conditions at $r=a$. That means $h$ has the form of

$$
\gamma_{m}=k_{0} \sin \theta_{0}+\frac{2 \pi m}{L_{0}}, \quad m=0, \pm 1, \pm 2, \ldots
$$

The generating function for scattering waves are there- fore

$$
\psi_{n m}^{(s)}=H_{n}^{(1)}(\rho) e^{j n \phi} e^{j \gamma_{m} z},
$$

with $\rho=r\left(k_{0}^{2}-\gamma_{m}^{2}\right)^{1 / 2}$. Expansion in terms of the vector harmonics yields

$$
\begin{aligned}
& \mathbf{E}_{s}=\sum_{n} \sum_{m}\left(a_{m}^{n} \mathbf{M}_{n m}^{(s)}+b_{m}^{n} \mathbf{N}_{n m}^{(s)}\right), \\
& \mathbf{H}_{s}=-j \sum_{n} \sum_{m}\left(a_{m}^{n} \mathbf{N}_{n m}^{(s)}+b_{m}^{n} \mathbf{M}_{n m}^{(s)}\right) .
\end{aligned}
$$

The expansion coefficients in Eq. (12) are to be determined by fitting the electromagnetic boundary conditions at the interface $r=a$ with the field configurations inside the region $r \leq a$. Since the system is piecewise homogeneous along the $z$ direction in the region $r \leq a$, the traditional solution approach would require additional boundarycondition fitting at periodic interfaces. However, that can be avoided by utilizing the periodicity in the problem to construct an eigenvalue problem ${ }^{18}$ and to obtain the corresponding eigenfields in analytic form. These eigenfunctions of the electromagnetic fields, which automatically satisfy all the internal interface boundary conditions, thus provide a basis for the expanding arbitrary field configurations in this region. The mathematical derivation for the eigenvalue equation, plus the explicit forms of the eigenfunctions, are given in Appendix $\mathbf{A}$.

For an incident plane wave with incident angle $\theta_{0}$ and wave vector $k_{0}$, the fields in the region $r \leq a$ can be written in terms of the eigenfields as

$$
\begin{aligned}
\mathbf{H}_{c} & =\sum_{n} \sum_{\mu}\left[\alpha_{\mu}^{n} \mathscr{H}_{h}\left(n, \Lambda_{\mu}^{h}\right)+\beta_{\mu}^{n} \mathcal{H}_{e}\left(n, \Lambda_{\mu}^{e}\right)\right], \\
\mathbf{E}_{c} & =\sum_{n} \sum_{\mu}\left[\alpha_{\mu}^{n} \mathscr{E}_{h}\left(n, \Lambda_{\mu}^{h}\right)+\beta_{\mu}^{n} \mathscr{E}_{e}\left(n, \Lambda_{\mu}^{e}\right)\right],
\end{aligned}
$$

where $\mu$ labels the eigenvalue and its associated eigenfield, $n$ is the azimuthal index, $\alpha_{\mu}^{n}$ and $\beta_{\mu}^{n}$ are expansion coefficients to be determined by fitting the boundary conditions at $r=a$, and $\Lambda_{\mu}^{h}$ and $\Lambda_{\mu}^{e}$ are the eigenvalues determined by the eigenvalue equation

$\cos \left(k_{0} L_{0} \sin \theta_{0}\right)-\cos \left(h_{2} L\right) \cos \left(h_{1} d\right)$

$$
+\frac{1}{2}\left[\tau \frac{h_{1}}{h_{2}}+\frac{1}{\tau} \frac{h_{2}}{h_{1}}\right] \sin \left(h_{2} L\right) \sin \left(h_{1} d\right)=0,
$$

in which $\tau=\epsilon$ for the TM mode $\left(H_{z}=0\right)$ and $\tau=1$ for the TE mode $\left(E_{z}=0\right)$ as indicated by the superscript (subscript) $h$ and $e$, respectively. $\mathscr{H}_{h}\left(n, \Lambda_{\mu}^{h}\right), \mathscr{E}_{h}\left(n, \Lambda_{\mu}^{h}\right)$ and $\mathscr{H}_{e}\left(n, \Lambda_{\mu}^{e}\right), \mathscr{E}_{e}\left(n, \Lambda_{\mu}^{e}\right)$ denote the eigenfunctions of the magnetic and the electric fields for the TM and the TE polarizations, respectively. Their analytic forms can be found in Appendix A, Eqs. (A15)-(A18).

The problem is now reduced to the determination of the expansion coefficients $a_{m}^{n}, b_{m}^{n}, \alpha_{\mu}^{n}$, and $\beta_{\mu}^{n}$. The boundary conditions at interface $r=a$ are

$$
\begin{aligned}
& E_{I \phi}+E_{s \phi}=E_{c \phi}, \\
& E_{I z}+E_{s z}=E_{c z}, \\
& H_{I \phi}+H_{s \phi}=H_{c \phi},
\end{aligned}
$$




$$
H_{I z}+H_{s z}=H_{c z} \text {. }
$$

Since all the functions of Eq. (15) have the same factor of $e^{j n \phi}$, it is clear that each $n$th component of Eq. (15) is decoupled from all the other components and therefore can be solved independently. Substituting Eqs. (9), (12), and (13) into Eq. (15), we obtain the $n$ th-component equations of Eq. (15). These equations can now be solved by expanding all the $z$-dependent functions in terms of the periodic Fourier basis $\exp \left(j 2 \pi m z / L_{0}\right)$. Multiplying both sides of these equations by $\exp \left(-j 2 \pi m z / L_{0}\right)$ and integrating $z$ over one period yields, after some algebraic rearrangements,

$$
\begin{aligned}
& a_{m}^{n}=\sum_{\mu} \Omega_{m \mu}^{n} \beta_{\mu}^{n}+\left(\widetilde{H}_{z}\right)_{m}^{n}, \\
& b_{m}^{n}=\sum_{\mu} \Psi_{m \mu}^{n} \alpha_{\mu}^{n}+\left(\widetilde{E}_{z}\right)_{m}^{n}, \\
& a_{m}^{n}+\Gamma_{m m}^{n} b_{m}^{n}=\sum_{\mu}\left(\Pi_{m \mu}^{n} \alpha_{\mu}^{n}+\Delta_{m \mu}^{n} \beta_{\mu}^{n}\right)=\left(\widetilde{E}_{\phi}\right)_{m}^{n}, \\
& \Gamma_{m m}^{n} a_{m}^{n}+b_{m}^{n}=\sum_{\mu}\left(\Theta_{m \mu}^{n} \alpha_{\mu}^{n}+\Xi_{m \mu}^{n} \beta_{\mu}^{n}\right)+\left(\widetilde{H}_{\phi}\right)_{m}^{n} .
\end{aligned}
$$

In Eq. (16), a tilde above the $E$ and $H$ denotes symbolically the resulting terms associated with incident waves after the integration over $z$ and the algebraic rearrangements. The explicit forms of all the matrix elements $\Omega_{m \mu}^{n}, \Psi_{m \mu}^{n}$, $\Gamma_{m m}^{n}, \Pi_{m \mu}^{n}, \Delta_{m \mu}^{n}, \Theta_{m \mu}^{n}, \Xi_{m \mu}^{n}, \widetilde{E}_{\phi m}^{n}, \widetilde{E}_{z m}^{n}, \widetilde{H}_{\phi m}^{n}$, and $\widetilde{H}_{z m}^{n}$ are given in Appendix B. Solution of Eq. (16) yields the expansion coefficients, and their substitution into Eqs. (12) and (13) gives the exact solution for the electromagnetic waves in the entire space.

\section{B. Absorption and scattering efficiency}

Various electromagnetic properties of the particle chain may be obtained from the rigorous scattering solution presented above. For instance, the scattering efficiency of the periodic particle chain is readily derived as

$$
\begin{aligned}
Q_{\text {sca }} & \equiv \frac{1}{2 a L_{0} I_{i}} \int_{0}^{L_{0}} \frac{1}{2} \operatorname{Re}\left(\mathbf{E}_{s} \times \mathbf{H}_{s}^{*}\right) \cdot \widehat{\mathbf{e}}_{r} a d \phi d z \\
& =\frac{2}{k_{0} a} \sum_{n} \sum_{|m| \leq m_{\max }}\left(\left|a_{m}^{n}\right|^{2}+\left|b_{m}^{n}\right|^{2}\right) \frac{1-\widetilde{\gamma}_{m}^{2}}{\cos ^{2} \theta_{0}},
\end{aligned}
$$

where $I_{i}$ is the intensity of the incident wave, $\widetilde{\gamma}_{m}^{2}=\left(\sin \theta_{0}+2 \pi m / k_{0} L_{0}\right)^{2}$, and $m_{\max }$ is the maximum integer that satisfies $\widetilde{\gamma}_{m}^{2} \leq 1$ for the scattering waves. ( $m>m_{\max }$ gives exponentially decaying evanescent waves.) In the derivation of Eq. (17) we have used the asymptotic forms of $\mathbf{E}_{s}$ and $\mathbf{H}_{s}$ at $r / a \gg 1$. The total extinction efficiency by definition is

$$
\begin{aligned}
Q_{\mathrm{ext}} & \equiv \frac{-1}{2 a L_{0} I_{i}} \int_{0}^{L_{0}} \frac{1}{2} \operatorname{Re}\left(\mathbf{E}_{I} \times \mathbf{H}_{s}^{*}+\mathbf{E}_{s} \times \mathbf{H}_{I}^{*}\right) \cdot \widehat{\mathrm{e}}_{r} a d \phi d z \\
& =\frac{2}{k_{0} a} \sum_{n} \operatorname{Re}\left(b_{0}^{n}\right)
\end{aligned}
$$

for the TM-polarized incident wave and

$$
Q_{\mathrm{ext}}=-\frac{2}{k_{0} a} \sum_{n} \operatorname{Im}\left(a_{0}^{n}\right)
$$

for the TE-polarized incident wave. From the definition of $Q_{\text {ext }}$, the absorption efficiency can be obtained from Eqs. (17) and Eq. (18) as

$$
Q_{\text {abs }}=Q_{\text {ext }}-Q_{\text {sca }} \text {. }
$$

One can also study the angle- or wave-vector-dependent scattering behaviors of the chain. In particular, the effect of high-multipole couplings on the depolarized scattering, defined as the component of the scattered waves with electric field perpendicular to that of the incident field vector, can be examined. In the next section, we numerically evaluate the characteristics of absorption and scattering from our model and compare them with experimental data.

\section{NUMERICAL RESULTS AND COMPARISON WITH EXPERIMENTS}

From experiments we set the parameters of our model as $L=150 \AA$ and $a=75 \AA$ corresponding to small spheres of diameter $150 \AA$ in the aggregates. ${ }^{9,10}$ The dielectric constant of the cylinder is obtained from the bulk dielectric constant of gold with the Drude collision time corrected due to the small particle size. ${ }^{19}$ The matrix is water with an optical index of refraction of 1.33. In our numerical calculations, we will concentrate on the properties in the optical frequencies.

The key step in our formulation is the construction of an eigenvalue problem for the field in $r \leq a$ to capture the electromagnetic coupling between the cylinders. The eigenvalues are given by the roots of Eq. (14) for both TM and TE polarizations. We first illustrate the properties of the eigenvalues by plotting in Fig. 2 the left-hand side of Eq. (14), denoted as $F$, as a function of $\Lambda^{2}$. Here the wavelength is taken to be $7200 \AA$, the separation distance between the adjacent cylinders is $150 \AA$, and the wave is assumed to be normally incident. We have omitted the small imaginary part in the dielectric constant for the purpose of illustration. The zero crossings of the curve represent the solutions of Eq. (14), i.e., eigenvalues. It is noted from the plot that there is always a maximum value for the real part of the eigenvalue. This enables us to order the eigenvalues according to the size of their real parts in the numerical calculation. The actual solutions of Eq. (14) in our calculation are obtained by a nonlinear-equation solver routine with accurate trial solutions provided by the method discussed in Appendix C. Linear combinations of the eigenfields give the field configurations excited in region $r \leq a$. We plot in Fig. 3 for the same parameters the function $Z_{i}(z)$ of Eq. (A7c) to illustrate the $z$ dependence of the eigenfunctions for the ground and excited states in both polarizations.

With the knowledge of the eigenvalues, the main task of the calculation is in the calculation of the matrix elements and the solution of Eq. (16). Since one cannot compute with infinite-order matrices, a reasonable cutoff value for the matrix order indices $m$ and $\mu$ is needed. In our case, a cutoff number for the order of the matrix is 
obtained when the expansion coefficients show indiscernable change by further increasing $m$ and $\mu$ in the calculation. We found that $m$ and $\mu \approx 15$ and $n=6$ are sufficient for an accurate result. It is helpful to note for this part of the calculation that when the argument of the Bessel and Henkel functions is large, it may pose problems for the accuracy for the matrix inversion. In this case, a redefinition (or scaling) of $a_{m}^{n}, b_{m}^{n}, \alpha_{\mu}^{n}$, and $\beta_{\mu}^{n}$ in Eq. (16) can make the problem well-posed again.

Plotted in Fig. 4 is the spectrum of absorption efficiency for different separation distances $d$ when a normally incident wave is polarized perpendicular to the chain axis (TE mode). In this case one expects the electromagnetic excitations in adjacent cylinders to be weakly coupled. Indeed, the absorption is peaked within a very narrow frequency band around $\lambda=5300 \AA$ which coincides with the Mie resonance for a single gold sphere. ${ }^{20}$ This indicates that in the TE mode each particle absorbs independently. However, when the incident wave has an electric field component along the chain axis as in the TM mode, the single-particle character may be

(a)

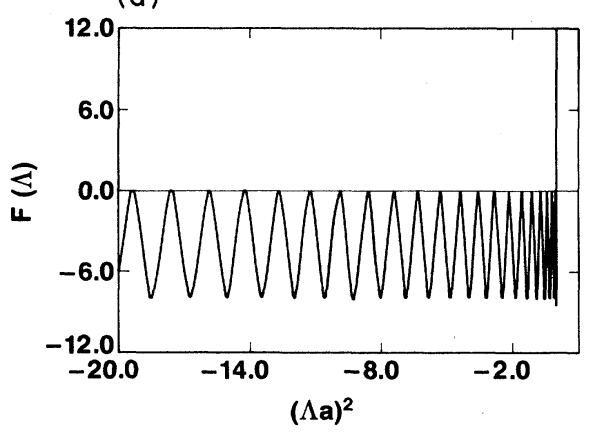

(b)

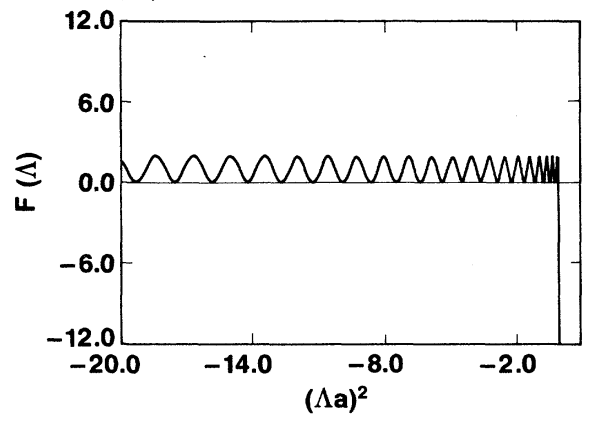

FIG. 2. (a) $F$ [left-hand side of Eq. (14) with $\tau=\epsilon$ ] vs the dimensionless separation constant $(\Lambda a)^{2}$ for the TM mode. Here $\lambda_{0}=7200 \AA, L=150 \AA, a=75 \AA$, and $d=150 \AA$. The zero crossings of $F$ represent the eigenvalues. The maximum eigenvalue in this case is $(\Lambda a)^{2}=\left(0.008944,0.4227 \times 10^{-4}\right)$, which corresponds to the lowest eigenmode. (b) $F$ [left-hand side of Eq. (14) with $\tau=1]$ vs the dimensionless separation constant $(\Lambda a)^{2}$ for the TE mode. Parameters are the same as those in the TM mode. The maximum eigenvalue in this case is $(\Lambda a)^{2}=(-0.03538,0.00246)$.

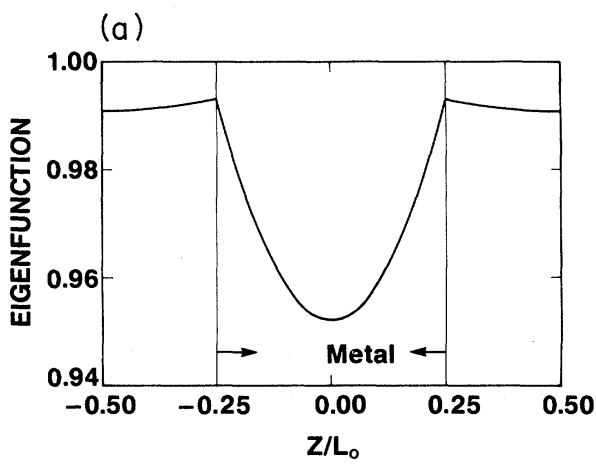

(b)

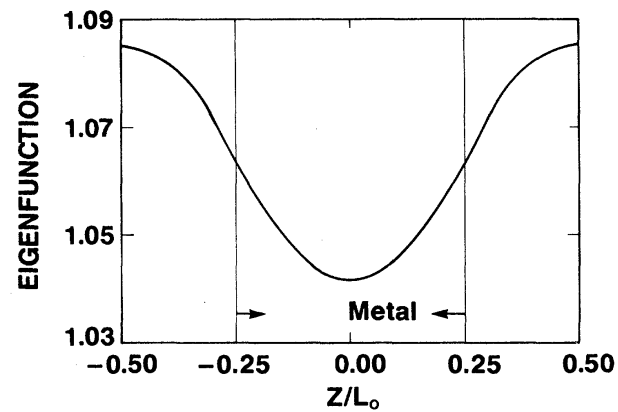

(c)

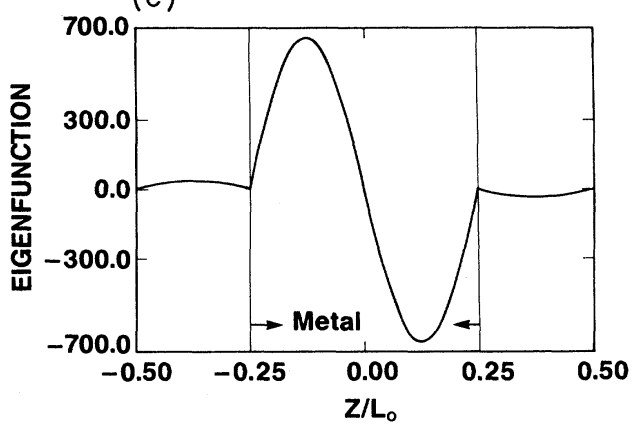

(d)

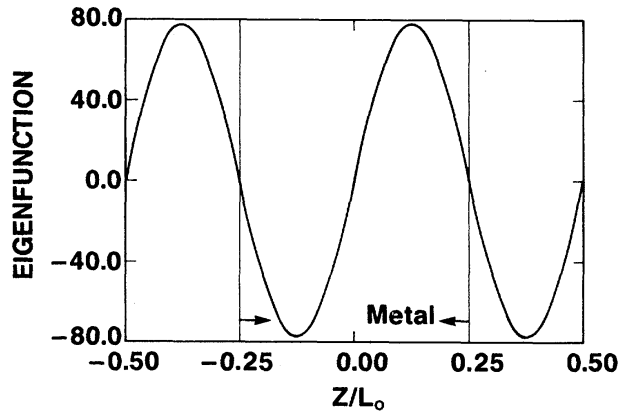

FIG. 3. (a) Plot of the ground-state eigenfunction $Z(z)$ in the TM mode. (b) Plot of the ground-state eigenfunction $Z(z)$ in the TE mode. (c) Plot of the excited eigenfunction $Z(z)$ in the TM mode. (d) Plot of the excited eigenfunction $Z(z)$ in the TE mode. 


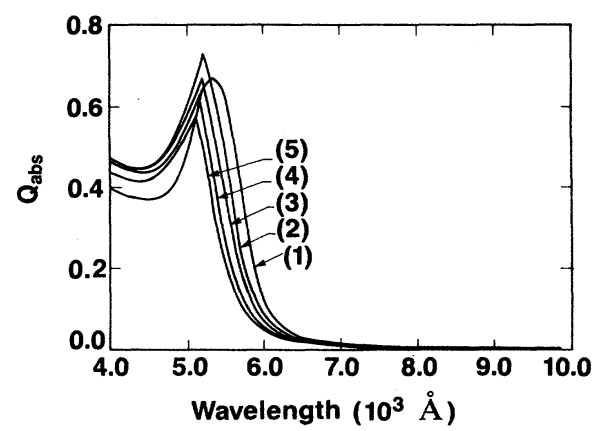

FIG. 4. Spectra of absorption efficiency for a normally incident wave with electric field polarized perpendicular to the chain axis. The absorption characteristics with different separation distances are similar, with a peak around $\lambda=5300 \AA$. The number above each curve corresponding to the separation distances as follows: curve $1, d=150 \AA$; curve $2, d=60 \AA$; curve $3, d=40 \AA$; curve $4, d=20 \AA$; curve $5, d=10 \AA$.

changed by the strong coupling of the electromagnetic fields between the adjacent cylinders. This is evident in the results shown in Fig. 5. For normal incidence, the component of the oscillating incident electric field along the chain axis induces a collective excitation at longer wavelength with a magnitude much larger than the single-particle peaks shown in Fig. 4. In fact, since $Q_{\text {abs }}$ can be interpreted as the ratio of the absorption cross section to the geometric cross section, it is seen that the induced collective excitation absorbs nearly a factor of 5-10 times the intensity incident on the geometric cross section. The collective excitation peak positions are noted to be strongly dependent on the separation distances between the adjacent cylinders. In Fig. 6 the peak frequency, normalized by the single-particle Mie-resonance frequency, is shown as a function of $d / L$. It is clear that when the cylinders are far apart, the collective resonance peak is positioned near the Mie-resonance peak, and as $d$ decreases it shifts to lower and lower frequencies and eventually disappears into $\omega=0$ when $d=0$, recovering



FIG. 5. Spectra of absorption efficiency for a normally incident wave with electric field polarized along the chain axis. The position of the low-frequency absorption peak is noted to be strongly dependent on the separation distance $d$ between adjacent cylinders. The value of $d$ associated with each curve is the same as those indicated in Fig. 4.

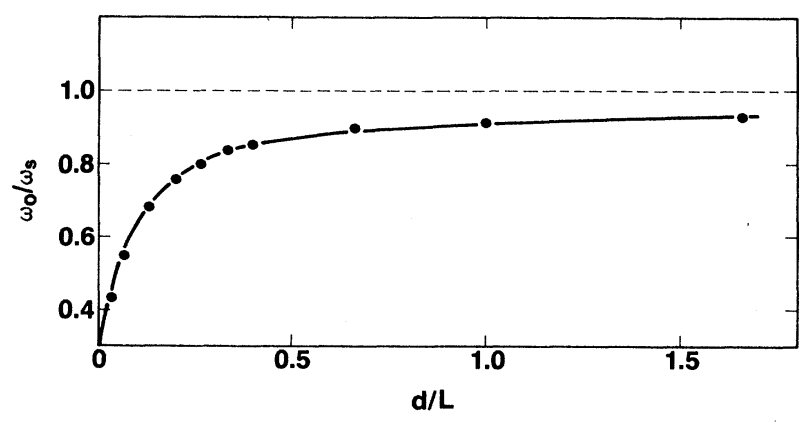

FIG. 6. The red-shifted absorption peak position $\omega_{0}$, normalized by the single-particle Mie-resonance frequency $\omega_{s}$, plotted against $d / L$ for a normally incident TM wave.

the solution for a homogeneous infinite cylinder. ${ }^{17}$ Since the incident wavelength in our problem is much larger than the particle sizes, the $m=0$ mode is dominant. We note, however, that while the red shift is predominantly a dipolar effect, yet the magnitude of the shift does depend on the sum of all the higher-order multipole interactions. Traditionally, the effect of these higher-order multipole

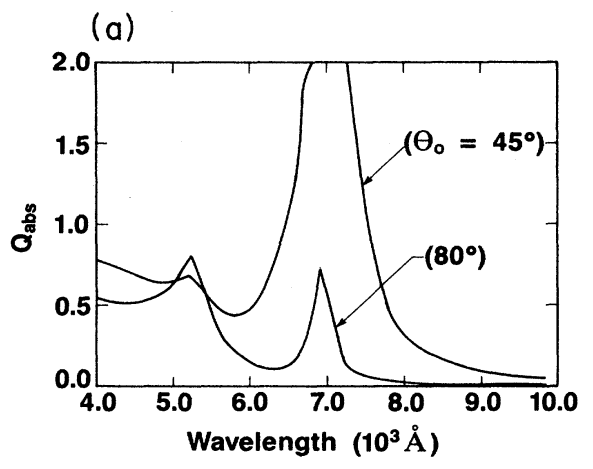

(b)

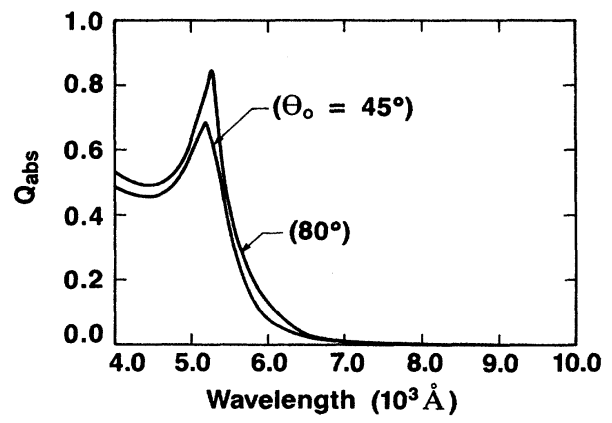

FIG. 7. (a) Spectra of absorption efficiency for a TM wave at two different incident angles. Two absorption peaks appear simultaneously in the spectra. When the incident angle is further away from the normal, the single-particle Mie-resonance peak becomes larger relative to the low-frequency excitation peak. (b) Spectra of absorption efficiency for a TE wave at two different incident angles. In contrast to the TM wave as shown in (a), only one absorption peak appears at the single-particle Mie-resonance frequency. 


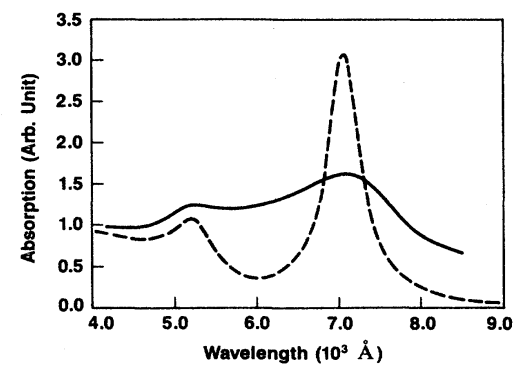

FIG. 8. Comparison of the calculated solid-angle-averaged absorption coefficient (dashed line) with the experimental result of the fractal gold aggregates. The calculated result has been normalized to the experimental result at $\lambda=4000 \AA$.

interactions has been equated to the appearance of multiple peaks in the absorption spectrum. Since this phenomenon is not apparent in our present calculation, we have performed separate calculations on tin particles where the multiple peaks have been obtained by Fuchs et al. ${ }^{12}$ Our results, presented in Appendix D, show that multiple peaks indeed appear in that case. We therefore conclude that the appearance or nonappearance of multiple peaks is also dependent on the dielectric constant and is not necessarily an indication of the presence or absence of multipole interactions. Another point we wish to note here is that the simplicity of our calculational method remains as one goes beyond the long-wavelength limit. Results of such calculations, however, will be reported elsewhere.

In Fig. 7(a), we show the absorption spectra for a TM wave incident at two different angles away from normal. The separation distance $d$ is set at $30 \AA$. It is clear that in both cases there are two peaks appearing simultaneously. By coupling this fact with the absorption characteristics of the TE wave incident at these angles as shown in Fig. $7(b)$, we conclude that when an aggregate cluster with locally chainlike structures is illuminated by an arbitrary incident wave, the absorption spectrum should display a double-peak character as seen experimentally. ${ }^{9}$ Plotted in Fig. 8 are the calculated solid-angle-averaged absorp-

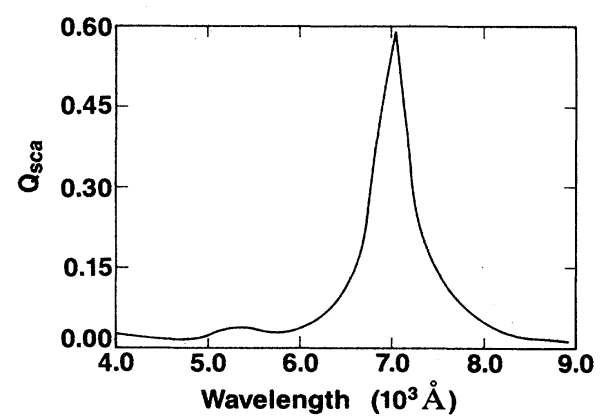

FIG. 9. Scattering efficiency plotted as a function of $\lambda$. The incident wave is polarized in the incident plane with the incident angle at $60^{\circ}$. The value of $d$ is set at $30 \AA$. The scattering is seen to be enhanced near the peak of the low-frequency excitation. tion efficiency, $\left\langle Q_{\mathrm{abs}}\right\rangle=\int Q_{\mathrm{abs}}\left(\theta_{0}, \phi_{0}\right) d \Omega_{0} / \int d \Omega_{0}$ (dashed line) and the experimental results (solid line). The value of $d$ is set at $24 \AA$, which is calculated by equating the capacitance $C=\epsilon_{0} \pi a^{2} / 4 \pi d$ for a capacitor of area $\pi a^{2}$ and separation $d$ with that of two touching spheres of radius $a$, given by $2 a \ln 2$. The agreement with the experimentally observed low-frequency absorption peak position is seen to be excellent. The overall agreement between the calculated and the experimental results is very good in view of the simplicity of our model. The difference in width and strength of the low-frequency peak is probably due to the finite chain length and its broad size distribution. Nevertheless, the appearance of the low-frequency absorption peak is a clear indication that the anisotropic multipole interaction plays an important role in the optical-absorption characteristics of aggregate clusters.

Now let us examine the scattering characteristics of our model. Figure 9 shows a typical spectrum of scattering efficiency. The incident wave is polarized along the chain axis with an incident angle $\theta_{0}=60^{\circ}$ and the separation distance $d=30 \AA$. Scattering is greatly enhanced at the low-frequency collective excitation frequency, in agreement with the experiment. However, the calculated result predicts a narrower width and stronger intensity than the experimental result, as expected. Since the optical extinction is the sum of the absorption and scattering, the above results thus imply that the optical extinction is greatly enhanced at this frequency due to the electromagnetic coupling between the cylinders.

Another directly observable manifestation of the collective excitation is the magnitude of the depolarized scattering, which can be characterized by means of a ratio of the depolarized scattering, $R=I_{V H} / I_{V V}$, where $I_{V H}$ $\left(I_{V V}\right)$ is the depolarized (polarized) scattering intensity. In Fig. 10 we compare the calculated $R$ (after solid-angle averaging) with the experiment. The magnitude of the calculated polarized scattering has been normalized to the value measured at $4880 \AA$. Here the $d$ is set again at $24 \AA$. Since the polarized scattering is highly wavevector dependent, the experimental data $^{7}$ are all mea-

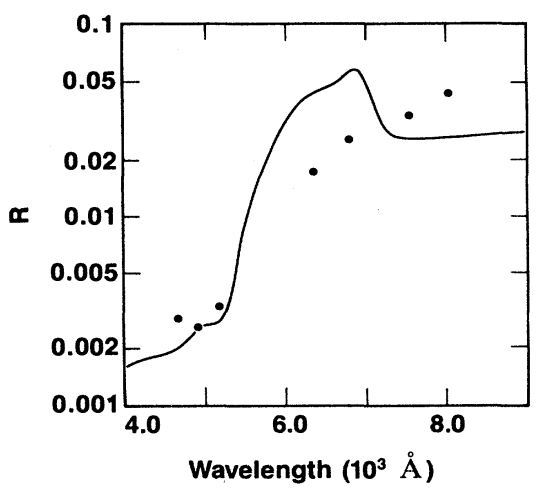

FIG. 10. Comparison of the depolarization ratio between the calculated (solid-angle-averaged) result and the experiment. The calculated and measured polarized scatterings are normalized at $\lambda=4880 \AA$. 
sured at the same $q=0.01 \mathrm{~nm}^{-1}$. The agreement with our theoretical calculation is very good, correctly accounting for the rise in $R$ as $I_{V V}$ increases due to the collective excitation.

\section{CONCLUDING REMARKS}

We have formulated a simple method for the rigorous solution of the electromagnetic scattering properties of a periodic chain of cylinders. The model allows a detailed study of the electromagnetic coupling between cylinders and its relationship to the geometric sizes and material compositions of the chain. Application of the model to the elucidation of optical characteristics of small goldparticle aggregates indicates that the second absorption peak as well as the enhanced depolarized scattering arise from high-multipole interaction between neighboring particles, with the position of the peak controlled by the strength of capacitive coupling. However, we note that the structure of the real aggregates is much more complicated than the simple periodic chain and accurate modeling of its optical behavior would require much more effort. Yet the rigorous solution of our simple model indicates what may result if one has the ability to control the precise microstructure and actually produce cylindrically segmented particles. One application would be the control over the position of the absorption peak which would be useful in infrared detection. In fact, further study in this direction has already revealed rich infrared and far-infrared absorption characteristics ${ }^{21}$ associated with variation in the geometric parameters.

\section{ACKNOWLEDGMENTS}

It is a pleasure to thank D. A. Weitz for first calling our attention to the optical data and for numerous helpful discussions during the course of this work. We also like to thank M. H. Lindsay and M. Y. Lin for discussions on the experimental results.

\section{APPENDIX A: EIGENFUNCTIONS OF ELECTROMAGNETIC FIELD IN THE REGION $r \leq a$}

In the region $r \leq a$, the system is piecewise homogeneous along the $z$ direction. We present in this appendix a simple method to construct an eigenvalue problem for the electromagnetic field, thus provide a unified basis of expansion for fields in this region.

We first write $\psi(r, \phi, z)=R(r) \Phi(\phi) Z(z)$. The three separated equations are

$$
\begin{aligned}
& \frac{\partial^{2} \Phi(\phi)}{\partial \phi^{2}}+n^{2} \Phi(\phi)=0 \\
& \frac{d^{2} Z(z)}{d z^{2}}+\left(k^{2}-\Lambda^{2}\right) Z(z)=0 \\
& \rho^{2} \frac{\partial^{2} R(\rho)}{\partial \rho^{2}}+\rho \frac{\partial R(\rho)}{\partial \rho}+\left(\rho^{2}-n^{2}\right) R(\rho)=0 .
\end{aligned}
$$

In Eq. (A3), $\rho=\Lambda r$. The first separation constant $n$ deter- mines the order of the Bessel function of Eq. (A3). The second separation constant $\Lambda$ is chosen such that it remains the same for different homogeneous regions, thus enabling us to construct an eigenvalue problem for the field. Since the fields must be finite at $r=0$, the generating function should be of the form

$$
\psi_{n}^{(e, o)}(r, \phi, z)=J_{n}(\Lambda r) e^{j n \phi}\left(\begin{array}{c}
\cos (h z) \\
\sin (h z)
\end{array}\right)
$$

where $h=\left(k^{2}-\Lambda^{2}\right)^{1 / 2}$, and the superscripts $(o)$ and $(e)$ denote the odd and even functions in the variable $z$, respectively. The corresponding vector harmonics are

$$
\mathbf{M}_{n}^{(e, o)}=\left[j n \Lambda \frac{J_{n}(\Lambda r)}{\Lambda r} \widehat{\mathbf{e}}_{r}-\Lambda J_{n}^{\prime}(\Lambda r) \widehat{\mathbf{e}}_{\phi}\right] e^{j n \phi}\left[\begin{array}{c}
\cos (h z) \\
\sin (h z)
\end{array}\right],
$$

and $\mathbf{N}_{n}=\boldsymbol{\nabla} \times \mathbf{M}_{n} / k$. It is helpful to note here that with the cylindrical symmetry, there are only two simple field configurations. One is the TM mode in which the $\mathbf{H}$ field is perpendicular to the chain axis, i.e., $H_{z}=0$, and the other is the TE mode in which the $\mathbf{E}$ field is perpendicular to the chain axis, i.e., $E_{z}=0$. In general, a field is expressible as a linear combination of both field configurations. In the following, we will discuss the TMmode field configuration in detail. Results for the TE mode are similar to that of TM mode and will be pointed out along with our discussions. For the TM mode, since the $\mathbf{N}$ function obtained from Eq. (A5) has a nonvanishing $z$ component, the appropriate vector harmonics for a general expansion of $\mathbf{H}$ must only be $\mathbf{M}_{n}$. That means

$$
\mathbf{H}_{n}^{i}=A_{n}^{i} \mathbf{M}_{n}^{(e)}(i)+B_{n}^{i} \mathbf{M}_{n}^{(o)}(i),
$$

where $A_{n}^{i}$ and $B_{n}^{i}$ are expansion coefficients to be determined later. Here $i$ is the index that labels the parameters and functions pertaining to the three homogeneous regions I, II, and III as shown in Fig. 1 in the text. Substituting Eq. (A5) into Eq. (A6) gives

$$
\begin{aligned}
\mathbf{H}_{n}^{i}= & \left(U_{n} \widehat{\mathbf{e}}_{r}-V_{n} \widehat{\mathbf{e}}_{\phi}\right) e^{j n \phi} Z_{i}\left(h_{i} z\right), \\
\mathbf{E}_{n}^{i}= & \frac{1}{-j k_{0} \epsilon_{i}} \nabla \times \mathbf{H}_{n}^{i} \\
= & \frac{-1}{j k_{0} \epsilon_{i}}\left[h_{i}\left(V_{n} \widehat{\mathbf{e}}_{r}+U_{n} \widehat{\mathbf{e}}_{\phi}\right) Z_{i}^{\prime}\left(h_{i} z\right)\right. \\
& \left.\quad+W_{n} Z_{i}\left(h_{i} \widehat{\mathbf{z}}\right) e_{z}\right] e^{j n \phi},
\end{aligned}
$$

where the terms with the $z$ variable are combined into a single function

$$
Z_{i}\left(h_{i} z\right)=A_{n}^{i} \cos \left(h_{i} z\right)+B_{n}^{i} \sin \left(h_{i} z\right),
$$

with

$$
U_{n}=j n \Lambda \frac{J_{n}(\rho)}{\rho}, \quad V_{n}=\Lambda \frac{d}{d \rho} J_{n}(\rho), \quad W_{n}=\Lambda^{2} J_{n}(\rho),
$$


and $h_{i}=\left(\epsilon_{i} k_{0}^{2}-\Lambda^{2}\right)^{1 / 2} . \quad Z_{i}^{\prime}$ in Eq. (A7b) denotes the derivative of $Z_{i}$ with respect to the argument $h_{i} z$.

With Eq. (A7), the transverse $(\phi, r)$ component of the fields can be arranged as a one-dimensional array in the order as shown in Eq. (A8). It is easy to verify that within each homogeneous region the value of the array at a plane $z=z$ is related to that at plane $z=z+l$ by a simple transfer matrix $T_{i}$, i.e.,

$$
\left(\begin{array}{c}
H_{n r}^{i}(z+l) \\
E_{n \phi}^{i}(z+l) \\
H_{n \phi}^{i}(z+l) \\
E_{n r}^{i}(z+l)
\end{array}\right)=T_{i}(l)\left(\begin{array}{c}
H_{n r}^{i}(z) \\
E_{n \phi}^{i}(z) \\
H_{n \phi}^{i}(z) \\
E_{n r}^{i}(z)
\end{array}\right),
$$

where the matrix $T_{i}(l)$ depends only on the separation distance $l$ of the two planes and is given by

$$
\left(\begin{array}{cccc}
\cos \left(h_{i} l\right) & -j \frac{k_{0} \epsilon_{i}}{h_{i}} \sin \left(h_{i} l\right) & 0 & 0 \\
-j \frac{h_{i}}{k_{0} \epsilon_{i}} \sin \left(h_{i} l\right) & \cos \left(h_{i} l\right) & 0 & 0 \\
0 & 0 & \cos \left(h_{i} l\right) & j \frac{k_{0} \epsilon_{i}}{h_{i}} \sin \left(h_{i} l\right) \\
0 & 0 & j \frac{h_{i}}{k_{0} \epsilon_{i}} \sin \left(h_{i} l\right) & \cos \left(h_{i} l\right)
\end{array}\right)
$$

The benefit of this construction is due to the fact that the transverse field at interfaces $z= \pm L / 2$ are continuous as required by the electromagnetic boundary conditions. Thus the transverse fields at different regions can be related to each other by a single matrix through the interface. Denoting the array in Eq. (A8) at the plane $z$ by $\mathbf{f}(z)$, we have

$$
\mathbf{f}\left(L_{0} / 2\right)=T_{\mathrm{III}}(d / 2) \times T_{\mathrm{II}}(L) \times T_{\mathrm{I}}(d / 2) \mathbf{f}\left(-L_{0} / 2\right) .
$$

In Eq. (A10) the distance between the two planes is one complete period and the periodicity requires that the field value at these two planes differ by only a phase factor of the incident wave, $e^{j k_{0} L_{0} \sin \theta_{0}}$, i.e.,

$$
\mathbf{f}\left(L_{0} / 2\right)=e^{j k_{0} L_{0} \sin \theta_{0}} \mathbf{f}\left(-L_{0} / 2\right)
$$

That means

$$
\left(T-I e^{j k_{0} L_{0} \sin \theta_{0}}\right) \mathbf{f}\left(-L_{0} / 2\right)=0,
$$

where we have denoted the product $T_{\mathrm{III}} \times T_{\mathrm{II}} \times T_{\mathrm{I}}$ in Eq.
(A10) by $T$ and the unit matrix by $I$. In order for Eq. (A12) to have a nontrivial solution, it is required that

$$
\operatorname{det}\left(T-I e^{j k_{0} L_{0} \sin \theta_{0}}\right)=0 \text {, }
$$

where det denotes the determinant. With a little algebra, Eq. (A13) can be rewritten in the following simple form:

$$
\begin{aligned}
& \cos \left(k_{0} L_{0} \sin \theta_{0}\right)-\cos \left(h_{2} L\right) \cos \left(h_{1} d\right) \\
& \quad+\frac{1}{2}\left[\epsilon \frac{h_{1}}{h_{2}}+\frac{1}{\epsilon} \frac{h_{2}}{h_{1}}\right] \sin \left(h_{2} L\right) \sin \left(h_{1} d\right)=0 .
\end{aligned}
$$

Since $h_{1}$ and $h_{2}$ contain the separation constant $\Lambda^{2}$, the solutions of Eq. (A14) determine the complete set of eigenvalues $\left\{\Lambda_{\mu}^{h}\right\}$, where the subscript $\mu$ labels the eigenvalues and the superscript $h$ denotes the TM mode. For each eigenvalue we obtain from Eq. (A12) a corresponding eigenvector $\mathbf{f}\left(-L_{0} / 2\right)$. The expansion coefficients $\left\{A_{n}^{i}(\mu), B_{n}^{i}(\mu)\right\}$ of Eq. (A6) are then obtained from this eigenvector through Eq. (A7). In their explicit form, the complete eigenfunctions of the TM mode are as follows:

$$
\begin{aligned}
& \mathscr{H}_{h}\left(n, \Lambda_{\mu}^{h}\right)=\left[j n \Lambda_{\mu}^{h} \frac{J_{n}\left(\Lambda_{\mu}^{h} r\right)}{\Lambda_{\mu}^{h} r} \widehat{\mathbf{e}}_{r}-\Lambda_{\mu}^{h} \frac{d}{d\left(\Lambda_{\mu}^{h} r\right)} J_{n}\left(\Lambda_{\mu}^{h} r\right) \widehat{\mathbf{e}}_{\phi}\right] e^{j n \phi} \\
& \times\left\{\begin{array}{l}
{\left[A_{n}^{\mathrm{I}}(\mu) \cos \left(h_{1} z\right)+B_{n}^{\mathrm{I}}(\mu) \sin \left(h_{1} z\right)\right] \quad\left(-L_{0} / 2 \leq z<-L / 2\right)} \\
{\left[A_{n}^{\mathrm{II}}(\mu) \cos \left(h_{2} z\right)+B_{n}^{\mathrm{II}}(\mu) \sin \left(h_{2} z\right)\right] \quad(|z| \leq L / 2),} \\
{\left[A_{n}^{\mathrm{III}}(\mu) \cos \left(h_{1} z\right)+B_{n}^{\mathrm{III}}(\mu) \sin \left(h_{1} z\right)\right] \quad\left(L / 2<z \leq L_{0} / 2\right)}
\end{array}\right. \\
& \mathscr{E}_{h}\left(n, \Lambda_{\mu}^{h}\right)= \begin{cases}\frac{1}{-j k_{0} \epsilon_{0}} \nabla \times \mathscr{H}_{h}\left(n, \Lambda_{\mu}^{h}\right) & (\text { in regions I, III ) } \\
\frac{1}{-j k_{0} \epsilon} \nabla \times \mathscr{H}_{h}\left(n, \Lambda_{\mu}^{h}\right) & \text { (in region II) } .\end{cases}
\end{aligned}
$$


In Eq. (A15),

$$
\begin{aligned}
& A_{n}^{\mathrm{I}}(\mu)=1, \\
& B_{n}^{\mathrm{I}}(\mu)=\frac{P_{1}}{e^{-j k_{0} L_{0} \sin \theta_{0}}-P_{2}}, \\
& A_{n}^{\mathrm{II}}(\mu)=P_{3}+P_{4} B_{n}^{\mathrm{I}(}(\mu), \\
& B_{n}^{\mathrm{II}}(\mu)=P_{5}+P_{6} B_{n}^{\mathrm{II}}(\mu), \\
& A_{n}^{\mathrm{III}}(\mu)=\left[\cos \left(h_{3} L_{0}\right)-\sin \left(h_{3} L_{0}\right) B_{n}^{\mathrm{I}}(\mu)\right] e^{j k_{0} L_{0} \sin \theta_{0}}, \\
& B_{n}^{\mathrm{III}}(\mu)=\left[\sin \left(h_{3} L_{0}\right)+\cos \left(h_{3} L_{0}\right) B_{n}^{\mathrm{I}}(\mu)\right] e^{j k_{0} L_{0} \sin \theta_{0}},
\end{aligned}
$$

(A16f)

where

$$
\begin{aligned}
P_{1}= & \cos \left(h_{2} L\right) \sin \left(h_{1} d\right) \\
& +\frac{h_{1} \epsilon}{h_{2}} \sin \left(h_{2} L\right) \sin \left(h_{1} L / 2\right) \sin \left[h_{1}\left(L_{0}-L / 2\right)\right] \\
& +\frac{h_{2}}{h_{1} \epsilon} \sin \left(h_{2} L\right) \cos \left(h_{1} L / 2\right) \cos \left[h_{1}\left(L_{0}-L / 2\right)\right],
\end{aligned}
$$

$$
\begin{aligned}
P_{2}= & \cos \left(h_{2} L\right) \cos \left(h_{1} d\right) \\
& +\frac{h_{1} \epsilon}{h_{2}} \sin \left(h_{2} L\right) \sin \left(h_{1} L / 2\right) \cos \left[h_{1}\left(L_{0}-L / 2\right)\right] \\
& -\frac{h_{2}}{h_{1} \epsilon} \sin \left(h_{2} L\right) \cos \left(h_{1} L / 2\right) \sin \left[h_{1}\left(L_{0}-L / 2\right)\right],
\end{aligned}
$$

$$
P_{3}=\cos \left(h_{1} L / 2\right) \cos \left(h_{2} L / 2\right)
$$$$
+\frac{h_{1} \epsilon}{h_{2}} \sin \left(h_{1} L / 2\right) \sin \left(h_{2} L / 2\right) \text {, }
$$

$$
\begin{aligned}
P_{4}= & -\sin \left(h_{1} L / 2\right) \cos \left(h_{2} L / 2\right) \\
& +\frac{h_{1} \epsilon}{h_{2}} \cos \left(h_{1} L / 2\right) \sin \left(h_{2} L / 2\right),
\end{aligned}
$$$$
P_{5}=-\cos \left(h_{1} L / 2\right) \sin \left(h_{2} L / 2\right)
$$$$
+\frac{h_{1} \epsilon}{h_{2}} \sin \left(h_{1} L / 2\right) \cos \left(h_{2} L / 2\right) \text {, }
$$

$P_{6}=\sin \left(h_{1} L / 2\right) \sin \left(h_{2} L / 2\right)$

$$
+\frac{h_{1} \epsilon}{h_{2}} \cos \left(h_{1} L / 2\right) \cos \left(h_{2} L / 2\right) \text {, }
$$

Applying the above procedure to the TE-mode case, we now expand the electric field in terms of $\mathbf{M}_{n}$ : and

$$
\mathbf{E}_{n}^{i}=C_{n}^{i} \mathbf{M}_{n}^{(e)}(i)+D_{n}^{i} \mathbf{M}_{n}^{(o)}(i),
$$

$$
\mathbf{H}_{n}^{i}=\frac{1}{j k_{0}} \nabla \times \mathbf{E}_{n}^{i} .
$$

By noticing the similarity of Eq. (A18) to Eq. (A7), we follow the same discussion for the TM mode and obtain an eigenvalue equation similar to Eq. (A14), except the explicit appearance of $\epsilon$ in Eq. (A14) is replaced by 1. By solving this equation, the eigenvalues $\Lambda_{\mu}^{e}$ and the corresponding expansion coefficients, $C_{n}^{i}(\mu)$ and $D_{n}^{i}(\mu)$, are obtained. The explicit form of these two expansion coefficients are in exact accord with the expansion coefficients $A_{n}^{i}(\mu)$ and $B_{n}^{i}(\mu)$ for the TM-mode case [Eqs. (A16) and Eq. (A17)], except the explicit appearance of $\epsilon$ is now replaced by 1 . The eigenfields for the TE mode are denoted by $\mathscr{E}_{e}\left(n, \Lambda_{\mu}^{e}\right)$ and $\mathscr{H}_{e}\left(n, \Lambda_{\mu}^{e}\right)$ in the text.

The actual field configuration excited by an incident wave can be a linear combination of $\left\{\mathscr{E}_{h}, \mathscr{H}_{h}\right\}$ and $\left\{\mathscr{E}_{e}, \mathscr{H}_{e}\right\}$. Therefore, in general, for $r \leq a$

$$
\begin{aligned}
& \mathbf{H}=\sum_{n} \sum_{\mu}\left[\alpha_{\mu}^{n} \mathscr{H}_{h}\left(n, \Lambda_{\mu}^{h}\right)+\beta_{\mu}^{n} \mathscr{H}_{e}\left(n, \Lambda_{\mu}^{e}\right)\right], \\
& \mathbf{E}=\sum_{n} \sum_{\mu}\left[\alpha_{\mu}^{n} \mathscr{E}_{h}\left(n, \Lambda_{\mu}^{h}\right)+\beta_{\mu}^{n} \mathscr{E}_{e}\left(n, \Lambda_{\mu}^{e}\right)\right],
\end{aligned}
$$

where $\alpha$ and $\beta$ are expansion coefficients to be determined by the boundary conditions fitting at $r=a$.

\section{APPENDIX B: MATRIX ELEMENTS}

The matrix elements of Eq. (16) are

$$
\begin{aligned}
& \Omega_{m \mu}^{n}=\left(\frac{\Lambda_{\mu}^{e}}{\widetilde{u}_{m} \eta}\right)^{2} \frac{J_{n}\left(\Lambda_{\mu}^{e}\right)}{H_{n}^{(1)}\left(\widetilde{u}_{m} \eta\right)} I_{m \mu}^{(a)}\left(\Lambda_{\mu}^{e}\right), \\
& \Psi_{m \mu}^{n}=j\left(\frac{\Lambda_{\mu}^{h}}{\widetilde{u}_{m} \eta}\right]^{2} \frac{J_{n}\left(\Lambda_{\mu}^{h}\right)}{H_{n}^{(1)}\left(\widetilde{u}_{m} \eta\right)} I_{m \mu}^{(b)}\left(\Lambda_{\mu}^{h}\right), \\
& \Pi_{m \mu}^{n}=\frac{n J_{n}\left(\Lambda_{\mu}^{h}\right)}{\widetilde{u}_{m} \eta H_{n}^{(1)^{\prime}}\left(\widetilde{u}_{m} \eta\right)} J_{m \mu}^{(b)}\left(\Lambda_{\mu}^{h}\right), \\
& \Delta_{m \mu}^{n}=\frac{\Lambda_{\mu}^{e} J_{n}^{\prime}\left(\Lambda_{\mu}^{e}\right)}{\widetilde{u}_{m} \eta H_{n}^{(1)^{\prime}}\left(\widetilde{u}_{m} \eta\right)} I_{m \mu}^{(a)}\left(\Lambda_{\mu}^{e}\right), \\
& \Theta_{m \mu}^{n}=j \frac{\Lambda_{\mu}^{h} J_{n}^{\prime}\left(\Lambda_{\mu}^{h}\right)}{\widetilde{u}_{m} \eta H_{n}^{(1)^{\prime}}\left(\widetilde{u}_{m} \eta\right)} I_{m \mu}^{(a)}\left(\Lambda_{\mu}^{h}\right), \\
& \Xi_{m \mu}^{n}=\frac{n J_{n}\left(\Lambda_{\mu}^{e}\right)}{j \widetilde{u}_{m} \eta H_{n}^{(1)^{\prime}}\left(\widetilde{u}_{m} \eta\right)} J_{m \mu}^{(a)}\left(\Lambda_{\mu}^{e}\right), \\
& \Gamma_{m m}^{n}=\frac{n H_{n}\left(\widetilde{u}_{m} \eta\right)}{\widetilde{u}_{m} \eta H_{n}^{(1)^{\prime}}\left(\widetilde{u}_{m} \eta\right)},
\end{aligned}
$$

and the corresponding components of incident waves for case (1), the TM mode, are

$$
\begin{aligned}
\left(\widetilde{E}_{Z}^{(1)}\right)_{m} & =-E_{n} \frac{J_{n}\left(\eta \cos \theta_{0}\right)}{H_{n}^{(1)}\left(\eta \cos \theta_{0}\right)} \delta_{m 0}, \\
\left(\widetilde{E}_{\phi}^{(1)}\right)_{m} & =-E_{n} \frac{n \sin \theta_{0} J_{n}\left(\eta \cos \theta_{0}\right)}{\eta \cos \theta_{0} H_{n}^{(1)^{\prime}}\left(\eta \cos \theta_{0}\right)} \delta_{m 0}, \\
\left(\widetilde{H}_{Z}^{(1)}\right)_{m} & =0, \\
\left(\widetilde{H}_{\phi}^{(1)}\right)_{m} & =-E_{n} \frac{J_{n}^{\prime}\left(\eta \cos \theta_{0}\right)}{H_{n}^{(1)^{\prime}}\left(\eta \cos \theta_{0}\right)} \delta_{m 0} .
\end{aligned}
$$


For case (2), the TE mode, we have

$$
\begin{aligned}
& \left(\widetilde{E}_{Z}^{(2)}\right)_{m}=0, \\
& \left(\widetilde{E}_{\phi}^{(2)}\right)_{m}=j E_{n} \frac{J_{n}^{\prime}\left(\eta \cos \theta_{0}\right)}{H_{n}^{(1)^{\prime}}\left(\eta \cos \theta_{0}\right)} \delta_{m 0}, \\
& \left(\widetilde{H}_{Z}^{(2)}\right)_{m}=j E_{n} \frac{J_{n}\left(\eta \cos \theta_{0}\right)}{H_{n}^{(1)}\left(\eta \cos \theta_{0}\right)} \delta_{m 0}, \\
& \left(\widetilde{H}_{\phi}^{(2)}\right)_{m}=j E_{n} \frac{n \sin \theta_{0} J_{n}\left(\eta \cos \theta_{0}\right)}{\eta \cos \theta_{0} H_{n}^{(1)^{\prime}}\left(\eta \cos \theta_{0}\right)} \delta_{m 0} .
\end{aligned}
$$

Here $\eta=k_{0} a, \quad \widetilde{\gamma}_{m}=\sin \theta_{0}+2 \pi m / k_{0} L_{0}$, and $\tilde{u}_{m}=(1$ $\left.-\widetilde{\gamma}_{m}^{2}\right)^{1 / 2}, \delta$ is the Kronecker delta function, $E_{n}$ is given by Eq. $(9 \mathrm{c})$ in the text, the prime denotes the derivative with the entire argument, and

$$
\begin{aligned}
& I_{m \mu}^{(a, b)}\left(\Lambda_{\mu}\right)=\frac{1}{L_{0}} \int_{0}^{L_{0}} \mathscr{F}^{(a, b)}\left(\Lambda_{\mu}\right) e^{-j k_{0} \tilde{\gamma}_{m} z} d z, \\
& J_{m \mu}^{(a, b)}\left(\Lambda_{\mu}\right)=\frac{1}{L_{0}} \int_{0}^{L_{0}} \frac{\partial \mathscr{F}^{(a, b)}\left(\Lambda_{\mu}\right)}{\partial z} e^{-j k_{0} \tilde{\gamma}_{m} z} d z,
\end{aligned}
$$

where $\mathscr{F}^{(a)}\left(\Lambda_{\mu}\right)$ corresponds to the function $Z_{i}$ of Eq. (A7c) for each homogeneous region along the $z$ direction and $\mathcal{F}^{(b)}\left(\Lambda_{\mu}\right)$ corresponds to $Z_{i} / \epsilon_{i}$ in the corresponding homogeneous region.

\section{APPENDIX C: TRIAL SOLUTIONS FOR THE EIGENVALUE EQUATION}

Maxwell's equations for the TM mode give two coupled equations relating $H_{\phi}$ and $E_{r}$ :

$$
\begin{aligned}
& \frac{\partial H_{\phi}}{\partial z}=j k_{0} \epsilon(z) E_{r}, \\
& \frac{\partial E_{r}}{\partial z}=j k_{0}\left(1-\frac{\Lambda^{2}}{\epsilon(z) k_{0}^{2}}\right) H_{\phi} .
\end{aligned}
$$

Similar equations hold between $H_{z}$ and $E_{\phi}$. The Fourier transform of the above equations yields

$$
\begin{aligned}
& \gamma_{m} \mathscr{H}_{m}=k_{0} \sum_{p} \epsilon_{m-p} \mathscr{E}_{p}, \\
& \gamma_{p} \mathscr{E}_{p}=\sum_{m}\left[k_{0} \delta_{p m} \mathscr{H}_{m}-\frac{\Lambda^{2}}{k_{0}} \sigma_{p-m} \mathscr{H}_{m}\right] .
\end{aligned}
$$

In Eq. (C2) $\mathcal{H}$ and $\mathscr{E}$ represent the Fourier components of the fields. Substituting Eq. (C2a) into Eq. (C2b), we have

$$
A X=\Lambda^{2} B X \text {, }
$$

where $X$ denotes the field components, $\Lambda^{2}$ is the separation constant, and the matrix elements of $A$ and $B$ are

$$
\begin{aligned}
A_{i j} & =k_{0}^{2} \frac{\epsilon_{i-j}}{\gamma_{j}}-\gamma_{j} \delta_{i j}, \\
B_{i j} & =\sum_{k=-\infty}^{\infty} \epsilon_{i}-k \frac{1}{\gamma_{k}} \sigma_{k-j},
\end{aligned}
$$

in which $\delta$ is the Kronecker delta function,

$$
\begin{aligned}
\epsilon_{k} & =\frac{1}{L_{0}} \int_{0}^{L_{0}} \epsilon(z) e^{j\left(2 \pi k / L_{0}\right) z} d z, \\
\sigma_{k} & =\frac{1}{L_{0}} \int_{0}^{L_{0}} \frac{1}{\epsilon(z)} e^{\left(2 \pi k / L_{0}\right) z} d z,
\end{aligned}
$$

and

$$
\gamma_{j}=k_{0} \sin \theta_{0}+\frac{2 \pi j}{L_{0}} .
$$

The solution of the eigenvalue equation (C3) would thus provide fairly accurate trial solutions to Eq. (14) in the text.

For the TE mode, we also have two coupled equations

$$
\begin{aligned}
& \frac{\partial E_{r}}{\partial z}=j k_{0} H_{\phi}, \\
& \frac{\partial H_{\phi}}{\partial z}=j k_{0}\left[\epsilon(z)-\frac{\Lambda^{2}}{k_{0}^{2}}\right] E_{r},
\end{aligned}
$$
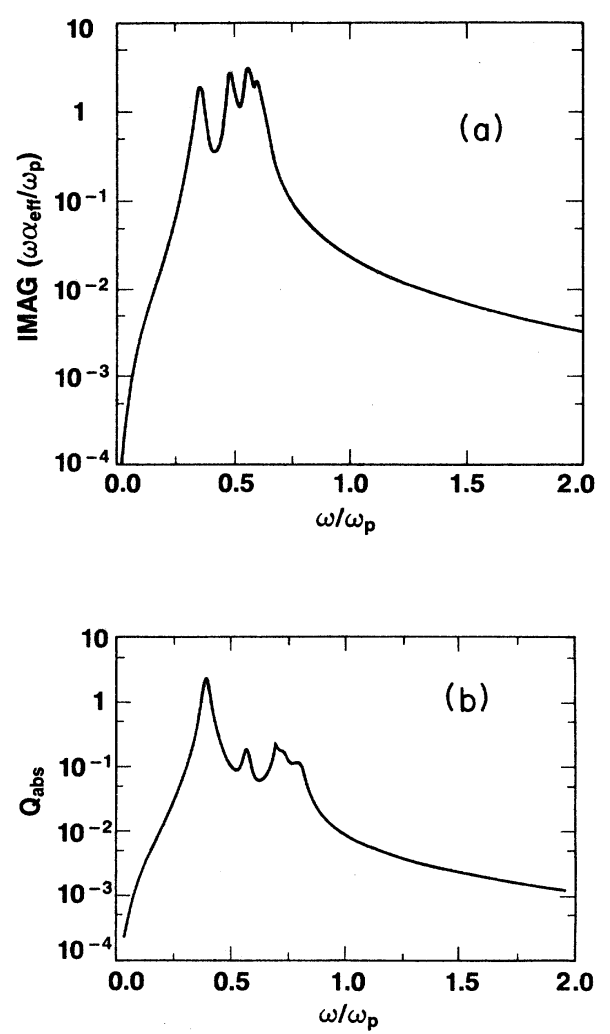

FIG. 11. Illustration of the effect of higher-order multipole interaction for tin particles. (a) gives a plot of the result by Fuchs et al. for two tin spheres. (b) is our result for a chain of tin particles. Parameters for both figures are discussed in Appendix D. The magnitude of the absorption plotted (a) and (b) is noted to differ by a calibration factor. Since (a) and (b) represent different physical systems, the comparison is for the purpose of seeing qualitative similarities. It is apparent from (b) that all the multiple peaks traditionally attributed to higherorder multipole interaction are reproduced in our calculation. 
and the equations between $E_{\phi}$ and $H_{r}$ are similar to Eq. (C7). The Fourier components of the field satisfy an equation similar to Eq. (C3) with

$$
\begin{aligned}
& A_{i j}=k_{0} \epsilon_{i-j}-\gamma_{j}^{2} \delta_{i j}, \\
& B_{i j}=\delta_{i j} .
\end{aligned}
$$

\section{APPENDIX D: EFFECT OF HIGHER-ORDER MULTIPOLE INTERACTIONS}

In this appendix, we present a comparison of our calculations on a chain of tin particles with that of Fuchs et $a l .{ }^{12}$ on two tin spheres in order to illustrate the effect of higher-order multipole interactions.

The parameters used in Ref. 12a are as follows: the radius of a sphere $a=30 \AA$, the separation between two centers of the spheres $D=61 \AA$, and the dielectric constant is given by Drude model with a plasmon frequency $\omega_{p}=1.17 \times 10^{16} \mathrm{sec}^{-1}$, Fermi velocity $v_{F}=1.24 \times 10^{8}$ $\mathrm{cm} / \mathrm{sec}$, and electron mean free path $l \sim a$. The main feature in the absorption spectrum that is attributed to multipole interaction is the presence of multiple peaks.

In our calculation, we use the same dielectric constant and set the radius of the cylinder at $30 \AA$ and the length of the cylinder at $60 \AA$. Since our model consists of a chain of particles, instead of two spheres, there cannot be an exact comparison. However, in Fig. 11 we show that if the particle separation is set at $30 \AA$, there is a very close qualitative correspondence with the calculated spectrum of Fuchs et al. ${ }^{12}$ In particular, the multiple peaks that have been attributed to multipole interaction have all been reproduced.
*Also at the Department of Physics, City College, City University of New York, New York, NY 10031.

${ }^{1}$ B. Donn (unpublished).

${ }^{2}$ E. L. Wright (unpublished).

${ }^{3}$ See, for example, Kinetics of Aggregation and Gelation, edited by F. Family and D. P. Landau (Elsevier, Amsterdam, 1984); D. A. Weitz and M. Oliveria, Phys. Rev. Lett. 52, 1433 (1984).

${ }^{4}$ M. Matsushita, K. Sumida, and Y. Sawada, J. Phys. Soc. Jpn. 54, 2786 (1985).

${ }^{5}$ D. A. Weitz, J. S. Huang, M. Y. Lin, and J. Sung, Phys. Rev. Lett. 54, 1416 (1985).

${ }^{6}$ D. W. Shaefer, J. E. Martin, P. Wiltzius, and D. S. Cannell, Phys. Rev. Lett. 52, 2371 (1984).

${ }^{7}$ Z. Chen, P. Sheng, D. A. Weitz, M. H. Lindsay, M. Y. Lin, and P. Meakin, Phys. Rev. B 37, 5232 (1988).

${ }^{8}$ R. Landauer, Electrical Transport and Optical Properties of Inhomogeneous Media (American Institute of Physics, New York, 1978), p. 2; D. Stroud and F. P. Pan, Phys. Rev. B 17, 1602 (1978).

${ }^{9}$ M. H. Lindsay, M. Y. Lin, D. A. Weitz, P. Sheng, Z. Chen, R. Klein, and P. Meakin, Faraday Discuss. Chem. Soc. 83, 153 (1987).

${ }^{10}$ D. A. Weitz, M. Y. Lin, J. S. Huang, T. A. Witten, S. K. Sinha, J. S. Gelhner, and R. C. Ball, in Scaling Phenomena in Disordered Systems, Vol. 133 of NATO Advanced Study Insti- tute Conference, Series B, edited by R. Pynn and A. Skjeltorp (Plenum, New York, 1985).

${ }^{11}$ T. Hollstein, U. Kreilbig, and F. Leis, Phys. Status Solidi B 82, 545 (1977).

${ }^{12}$ R. Fuchs and F. Claro, Phys. Rev. B 35, 3722 (1987); F. Claro, ibid. 30, 4989 (1984), and references therein.

13J. M. Gerardy and M. Ausloos, Phys. Rev. B 22, 4950 (1980), and references therein.

${ }^{14}$ P. Clippe, E. Evrard, and A. A. Lucas, Phys. Rev. B 14, 1715 (1976).

${ }^{15}$ U. Kreibig, A. Althoff, and M. Pressmann, Surf. Sci. 106, 308 (1981); M. Quinten and U. Kreibig, ibid. 172, 557 (1986).

${ }^{16} \mathrm{~K}$. Ohtaka and M. Inoue, Phys. Rev. B 19, 5057 (1979); 25, 689 (1982); 26, 3487 (1982); J. Phys. Jpn. 52, 1457 (1982).

${ }^{17}$ See, for example, C. F. Bohren and D. R. Huffman, Absorption and Scattering of Light by Small Particles (Wiley, New York, 1983).

${ }^{18}$ P. Sheng, R. S. Stepleman, and P. N. Sanda, Phys. Rev. B 26, 2907 (1982).

${ }^{19}$ R. W. Cohen, G. D. Cody, M. D. Coutts, and B. Abeles, Phys. Rev. B 8, 3689 (1973).

${ }^{20}$ For particle diameter between 50 and $200 \AA$, the Mieresonance position is relatively independent of the particle size and is determined by the Frölich condition of $\epsilon+2 \epsilon_{0}=0$; see also Ref. 14.

${ }^{21}$ M. Y. Zhou, P. Sheng, Z. Chen, and S. T. Chui (unpublished). 